\title{
LIBERTÀ E CONTINGENZA DELLA FRUIZIONE BEATIFICA NELLO SCOTISMO DEL PRIMO '300
}

Guido Alliney*

SÍNTESE - Este estudo tem como objeto a recepção da teoria scotista da vontade no início do século 14. Interesse precípuo é o modo como autores, sobretudo franciscanos, a partir das Universidades de Paris e de Oxford, discutiram sobre a possibilidade de uma escolha livre ou de um ato da própria vontade, por parte dos bemaventurados, quando da visão de Deus. Para tanto, pressuposições gerais da teoria scotista da vontade são apresentadas, bem como as inovações dos filósofos influenciados por Scotus.

PALAVRAS-CHAVE - Teoria scotista da vontade Visão beatífica. Liberdade. Influência do pensamento scotista no século 14
ABSTRACT - This study aims to analyse the reception of Scotus's theory of will in the beginning of the $14^{\text {th }}$ Century. The main interest is the way some authors, specially Franciscan thinkers, departing from the Universities of Paris and Oxford, discussed about the possibility for the blessed of a free choice or an act of the will itself concerning the vision of God. For this purpose, general pressupositions of Scotus's theory of will shall be presented, as well as the innovations of those philosophers influenced by Scotus.

KEY WORDS - Scotus's theory of will. Beatific vision. Freedom. Influence os Scotistic thought in the $14^{\text {th }}$ Century.

\section{La teoria scotiana della volontà}

La teoria della volontà di Duns Scoto $^{1}$ è contrassegnata da un forte carattere di novità, subito percepito dagli stessi teologi contemporanei. L'attenzione di questi

\footnotetext{
Università di Triste.

Questo lavoro espone in maniera sintetica i risultati di miei precedenti studi: G. Alliney, "L'autodeterminazione della volontà nella fruizione beatifica secondo Roberto Cowton e Riccardo Drayton", Esercizi filosofici 1 (1992), pp. 73-108; G. Alliney, "E necessario amare Dio? Una questione inedita di Guglielmo di Alnwick sulla fruizione beatifica" (con l'edizione di Guillelmus de Alnwick, In Sent., I, d. 1, q. 1, Utrum necesse sit voluntatem frui ultimo fine sibi ostenso a ratione), in G. Alliney, L. Cova (eds.), Parva Mediaevalia. Studi per Maria Elena Reina, Trieste 1993, pp. 87-128; G. Alliney, "Fra Scoto e Ockham: Giovanni di Reading e il dibattito sulla libertà a Oxford (13101320)" (con l'edizione di Ioannes de Reading, In Sent., I, d. 1, q. 6, Utrum fine ultimo apprehenso ab intellectu creato voluntas necessario velit illum finem), Documenti e studi sulla tradizione filosofica medievale VII (1996), pp. 243-368; G. Alliney, "La ricezione della teoria scotiana della volontà nell'ambiente teologico parigino (1307-1316)" (con l'edizione di Hugo di Novocastro, In Sent. I, d. 1, q. 3, Utrum ultimo fine apprehenso in universali in via, voluntas necessario feratur in illum et velit ipsum), Documenti e studi sulla tradizione filosofica medievale XVI (2005), in corso di stampa; G.
}

\begin{tabular}{|l|l|l|l|l|l|}
\hline VERITAS & Porto Alegre & v. 50 & n. 3 & Setembro 2005 & p. 95-108 \\
\hline
\end{tabular}


ultimi si focalizzò sull'esito più rivoluzionario, per il quale lo stesso beato di fronte alla visione di Dio potrebbe decidere di non esercitare alcun atto della propria volontà nei suoi confronti. Mai, negli accesi dibattiti degli ultimi decenni del XIII secolo, le posizioni dei teologi inseriti nella tradizione agostiniana e bonaventuriana erano state così estreme $e^{2}$. Anche se vi era stato chi avevano prospettato un'ipotesi simile, come Pietro di Giovanni Olivi o Gonsalvo Ispano, maestro dello stesso Scoto, nessuno aveva difeso con una tale solidità argomentativa questa conclusione, collocandola al termine di un complesso e coerente percorso filosofico. Per poter seguire la fortuna della dottrina scotiana della volontà, è allora innanzitutto opportuno esporne i nuclei teorici qualificanti.

A. Natura e volontà: Un agente naturale e un agente libero sono costitutivamente diversi, dato che ciascuno di essi ha un modo intrinseco di agire non riducibile all'altro. Questa opposizione non distingue l'ambito meramente operativo dei principi coinvolti, ma caratterizza invece a livello metafisico le potenze attive: natura e libertà rappresentano una coppia di trascendentali disgiuntivi differenti già nelle rispettive ragioni formali.

Un agente naturale esercita il proprio atto sempre al massimo delle proprie possibilità per una forma intrinseca che lo spinge a tale azione, e dunque agisce con necessità; un agente libero agisce invece spontaneamente in base alle proprie motivazioni. Anche l'intelletto è una potenza naturale, poiché dà sempre e con necessità il proprio assenso ai principi scientifici e alle conclusioni sillogistiche da essi guadagnati.

L'unico principio attivo libero è la volontà, potenza razionale autodeterminata capace di agire contingentemente. La volontà, infatti, non è necessitata ad agire dalla presenza dell'oggetto, come accade alle potenze naturali, ma si determina in base a motivi puramente intrinseci. La volontà in quanto libera è perciò superiore ad ogni potenza naturale, e ha quindi potere e controllo sulla loro attività, e in particolare su quella dell'intelletto.

B. Volontà e libertà: La volontà è costitutivamente libera; la libertà compete solo alla volontà. Ė l'esito della precedente divisione fra agenti naturali e liberi: la libertà è la caratteristica formale della volontà, per cui non si dà libertà senza volontà, né volontà senza libertà.

La libertà non implica la contingenza. La libertà qualifica a livello essenziale una potenza, mentre la contingenza caratterizza intrinsecamente le azioni della potenza che possono essere prodotte altrimenti, o non essere prodotte. In altre parole, mentre "libero" è il modo di essere della volontà, "necessario" o "contin-

Alliney, "The Treatise on the Human Will in the Collationes oxonienses attributed to John Duns Scotus" (con l'edizione di Ioannes Duns Scotus, Collationes Oxonienses, qq. 18-23), Medioevo XXX (2005), in corso di stampa.

2 Lo stesso Enrico di Gand, rappresentante del volontarismo più avanzato del XIII secolo, aveva sostenuto la necessità della fruizione non solo del beato, ma già del viator nei confronti del concetto di bene, ammettendo la contingenza dell'atto volontario unicamente in riferimento al fine conosciuto oscuramente, cioè Dio colto per fede o per astrazione. 
gente" indicano il modo di agire della potenza. Per questo motivo, non è conseguente che una potenza libera agisca contingentemente: il passaggio richiede una giustificazione metafisica.

Il concetto di "libertà" si predica in maniera univoca di Dio e dell'uomo. Per Scoto anche la volontà in quanto perfezione dell'essere è un trascendentale: questo significa che la definizione formale della volontà si pone precedentemente alla distinzione modale fra essere finito ed essere infinito, e deve essere comune ai due modi dell'essere. Mentre in Dio la volontà si mantiene al livello di perfezione pura, nella creatura ciò non accade: la volontà umana dunque è libera come quella divina, ma non altrettanto perfetta.

C. Libertà e contingenza: Libertà e contingenza fanno riferimento ad ambiti diversi della volontà che non devono essere confusi. Al livello più alto di perfezione la libertà della volontà implica la necessità del volere, come accade nell'attività produttiva essenziale di Dio: lo Spirito Santo è spirato con necessità assoluta, ma anche con perfetta libertà. Perché la volontà possa però mantenersi a tale livello di perfezione pura è necessario che sia il soggetto sia l'oggetto dell'atto volitivo siano perfetti, e ciò accade solo nell'azione divina ad intra. In Dio la perfezione implica l'infinità, la quale rende realmente identici e solo formalmente distinti gli attributi divini.

Se si esce dall'ambito della modalità infinita dell'essere la necessità cessa di essere una caratteristica della volontà. Già l'azione divina ad extra, cioè la creazione, è contingente perché rivolta ad enti che sono costitutivamente contingenti in quanto non esistenti per se stessi, ma portati all'essere proprio dall'atto volitivo divino.

Da questa metafisica ontologica segue che la volontà creata, caratterizzata da un livello deficitario di essere rispetto a quella divina, non può mai elevarsi a livello di perfezione pura, e perciò agisce sempre contingentemente. Tuttavia la differenza modale fa sì che, a livello degli enti finiti, la contingenza sia una perfezione rispetto alla necessità, dato che questa non potrebbe essere la necessità assoluta della produzione trinitaria, ma la mera necessità naturale.

La teoria della volontà di Scoto si struttura dunque in una serie ascendente di piani dottrinali: un primo livello distingue i principi naturali dai liberi, opponendo così l'intelletto alla volontà; un secondo livello stabilisce una definizione univoca di libertà comune a Dio e alla creatura; un terzo e ultimo collega infine la libertà creaturale con la contingenza nell'agire.

\section{La ricezione della teoria scotiana della volontà}

La dottrina sviluppata da Duns Scoto in merito alla libertà della volontà creata, come si è visto, comporta rilevanti considerazioni di ordine metafisico (in merito alla differenza costitutiva fra "natura" e "libertà", e ai modi in cui l'essere si manifesta, ovvero il finito e l'infinito) per giungere ad una conclusione certamente innovativa: la contingenza della fruizione beatifica in patria. Tuttavia, a dispetto di 
ciò che la storiografia spesso ha ameno implicitamente ammesso, questo esito della riflessione scotiana non è accolto con il previsto entusiasmo né nel milieu francescano parigino, né in quello oxoniense. In questa seconda parte dello studio si ricostruirà sinteticamente l'impatto della lezione scotiana sui teologi francescani dell'inizio del XIV secolo.

\subsection{Parigi}

È noto come Parigi si dimostrò un ambiente più favorevole di altri alle novità del pensiero di Scoto. Questo risultato generale non deve però essere applicato in maniera acritica anche al tema qui in esame. Come si è detto nella prima sezione, il pensiero di Scoto si articola su livelli dottrinali diversi e successivi; ora, solo i primi due livelli sembrano generalmente accolti dai teologi francescani parigini, mentre maggiori resistenze si incontrano riguardo all'esito finale, che spesso è rifiutato a vantaggio della più moderata conclusione per la quale la perfezione dell'oggetto rende necessaria l'azione volontaria del beato nei confronti dell'Essenza divina.

Quando Duns Scoto parte per Colonia nel 1307, Alessandro d'Alessandria prende il suo posto come maestro reggente in teologia nello studio francescano di Parigi $^{3}$. Alessandro, nel suo Quodlibet composto prima del 1310, affronta la questione della necessità della volizione nei confronti del fine ultimo rifiutando, al pari di Scoto, ogni naturalità della necessità del volere poiché natura e volontà sono principi opposti che non possono coesistere. Alessandro ammette la necessità della fruizione solo in patria, al cospetto cioè della visione chiara dell'Essenza divina, ma reputa che tale necessità non derivi dalla naturalità della volontà, né le sia intrinseca, ma nasca invece spontaneamente nella volontà stessa per l'adeguatezza dell'oggetto presentato. ${ }^{4}$ Un ulteriore approfondimento è offerto da Alessandro trattando della questione se lo Spirito Santo sia prodotto per mezzo della volontà, e soprattutto in che accezione la volontà possa esserne il principio produttivo. Il teologo esclude che si tratti della volontà in communi, perché in tal caso ogni

\footnotetext{
Su Alessandro d'Alessandria, così come per gli altri autori citati in seguito, si veda l'utile studio di R.L. Friedman, "The Sentences Commentary, 1250-1320. General Trends, the Impact of the Religious Orders, and the Test Case of Predestination", in G. Evans (ed.), Mediaeval Commentaries on the Sentences of Peter Lombard, Brill 2002, pp. 42-128: pp. 74-75. Per maggiori elementi bibliografici e notizie sulla tradizione manoscritta, cf. M. Rossini, C. Schabel, "Time and Eternity among the Early Scotists. Texts on Future Contingents by Alexander of Alessandria, Radulphus Brito, and Hugh of Novocastro", Documenti e studi sulla tradizione filosofica medievale XVI (2005), in corso di stampa.

4 "Sed hoc (scil. necessariam productionem actus) habet voluntas respectu talis obiecti quod est sibi adequatum, non quidem per naturam, sed sicut natura habet suo modo necessitatem annexam, ita voluntas habet <necessitatem> respectu talis obiecti. Que talis necessitas non excludit ab ea libertatem, quia respectu talis obiecti libere velle et necessario velle non opponuntur" (Alexander de Alexandria, Quodlibet, q. 16, citato in Alliney, "La ricezione", testo di nota 12).
} 
volontà potrebbe produrre lo Spirito Santo. Si tratta, invece, della volontà in quanto natura divina, formalmente distinta dalle altre perfezioni essenziali. ${ }^{5}$

Alessandro si mostra dunque a conoscenza delle dottrine scotiane, e ne impiega due elementi fondamentali: l'opposizione fra natura e volontà; e la concezione della volontà come trascendentale, perfezione assoluta comune a Dio e alla creatura. Tuttavia la sua dottrina non si può dire vicina a quella di Scoto, dato che l'univocità è intesa come unità reale: Alessandro non considera la differenza modale come intimamente connessa alla concezione trascendentale delle perfezioni pure. Limitare poi la contingenza della volontà allo stato del viator rende la posizione di Alessandro più simile a quella di Enrico di Gand che a quella radicale del Dottor Sottile.

Tutto ciò fa pensare più ad impiego di materiale dottrinale di provenienza scotiana in circolazione nell'ambiente francescano parigino piuttosto che ad una adesione precisa alla dottrina di Scoto.

Per renderci meglio conto della differenza fra una generica influenza della terminologia scotiana e un impiego teoreticamente fondato delle dottrine di Scoto vale esaminare la posizione di Ugo di Novocastro. Poiché probabilmente è il primo francescano legato a Scoto che legge le Sentenze a Parigi, ad Ugo è ormai ampiamente riconosciuta una notevole influenza nello sviluppo del primo scotismo in Francia. ${ }^{6}$

In I Sent. d. 10 q. 2, Ugo riporta condividendola l'opinione di Scoto per cui natura e volontà sono principi opposti, così che la necessità naturale e la necessità libera sono irriducibilmente distinte. Ugo fa poi un uso corretto della concezione della volontà come trascendentale passibile di una definizione univoca quando dimostra che produrre con necessità non ripugna alla volontà divina nella spirazione dello Spirito Santo. Ugo spiega infatti che il produrre necessariamente rappresenta la perfezione assoluta della volontà che compete alla volontà divina appunto in quanto infinita: ${ }^{7}$ la definizione formale della volontà in communi indica una adesione esplicita e coerente di Ugo ai principi cardine della dottrina del maestro.

\footnotetext{
"Quero enim secundum quam rationem voluntas est principium productivum Spiritus Sancti: non enim voluntas in communi, aliter omnis voluntas posset producere Spiritum Sanctum [... luntas ut natura divina magis est ratio principiandi quam voluntas" (Alexander de Alexandria, Ouodlibet, q. 16, citato in Alliney, "La ricezione", testo di nota 13).

$6 \quad$ Su Ugo di Novocastro si vedano L. Amorós, "Hugo von Novocastro O.F.M. und sein Kommentar zum ersten Buch der Sentenzen", Franziskanische Studien 20 (1933), pp. 177-222, e V. Heynck, "Der Skotist Hugo de Novo Castro OFM", Franziskanische Studien 43 (1961), pp. 244-70. Tradizionalmente la data di composizione del Commento di Ugo si colloca fra il 1307 e il 1317, ma Heynk restringe l'intervallo ai primi anni del secondo decennio del secolo. Da qui l'importanza del commentario, che precede quelli dei più noti scotisti come Giovanni di Bassoles, Guglielmo di Alnwick e Francesco di Meyronnes. Il più significativo studio dottrinale su Ugo di Novocastro resta E. Randi, Il sovrano e l'orologiaio. Due immagini di Dio nel dibattito sulla "potentia absoluta" fra XIII e XIV secolo, Firenze 1987, con edizione delle distinzioni $42-44$ del primo libro sulle Sentenze. Ma si veda anche Rossini, Schabel, "Time and Eternity among the Early Scotists".

Hugo de Novocastro, In Sent., I, d. 10, q. 2, ed. W. Volz, in Id., Die Lehre des Johannes de Bassolis von den Produktionen in Gott. Ein Vergleich mit der Lehre des Johannes Duns Scotus, München 1969, p. 252, nota 32.
} 
Nella questione dedicata alla modalità della fruizione Ugo, dopo aver riferito la dottrina, a suo parere maggiormente condivisa, di chi sostiene la necessità in via della volizione del fine ultimo in generale, ma non in particolare, ${ }^{8}$ espone la posizione di Scoto, che egli giudica nuova, per poi dichiarare esplicitamente di aderire a tale dottrina, e ribattere agli argomenti a sostegno dell'altra opinione. ${ }^{9}$

Se questo può sembrare l'atteggiamento di un discepolo devoto, va però notato che Ugo sorprendentemente limita il dibattito allo status viae, ${ }^{10}$ e non accenna neppure alla modalità della fruizione in patria, che per Scoto, come è noto, è contingente quanto quella in via per l'assoluta intrinsecità dell'azione volontaria. Ugo lascia a noi il compito di interpretare il suo silenzio, che può forse essere letto come una prudente espressione di un dissenso dagli esiti più estremi del volontarismo di Scoto.

Giacomo d'Ascoli, maestro reggente dello studio francescano parigino nel 1310-1311, dopo Nicolò di Lira discute delle questioni quodlibetali durante la reggenza, probabilmente nel 1311. ${ }^{11}$ Alla nostra ricerca interessa in particolare il secondo articolo della questione dove Giacomo, allo scopo di porre una distinzione ex natura rei nella semplicità divina, vuole dimostrare che intelletto e volontà in Dio non coincidono, ed analizza perciò i modi di agire della natura e della volontà in generale per distinguerli essenzialmente con una distinzione che sia valida tanto in Dio quanto nelle creature. Il teologo attribuisce alla natura il modo di agire naturale e necessario, ma poco opportunamente opera un confronto con la volontà in rapporto ad oggetti particolari - i beni intermedi senza una necessaria connessione con il fine ultimo - e di conseguenza assegna alla volontà in generale il modus agendi libero e contingente. ${ }^{12}$ Si tratta di una conclusione paradossale perché, anche se in accordo con Scoto è vero che nei confronti dei beni ordinati al fine sia la volontà divina sia quella creata agiscono libere et contingenter, questo non è ovviamente imputabile al concetto di volontà in communi, cioè al concetto trascendentale di volontà precedente alla differenza modale. Giacomo d'Ascoli sembra sovrapporre libertà e contingenza ad un livello improprio che lo porta al travisare l'univocità trascendentale scotiana in un'unità reale.

Purtroppo il Commento di Giacomo d'Ascoli non ci è giunto, ${ }^{13}$ per cui non possiamo integrare questo breve passo con altri testi. Possiamo però evidenziare

8 Scoto aveva giudicato contradditoria tale opinione sostenuta da Enrico di Gand.

"Alia est nova opinio Scoti, qui ponit contrarium ad hoc (scil. quod fine ultimo apprehenso in universali in via voluntas necessario vult). [...] Hoc tenendo, dico ad argumenta alterius opinionis" (Hugo de Novocastro, In Sent., I, d. 1, q. 3, citato in Alliney, "La ricezione", App. A, nn. 12-13).

${ }_{10}$ Come si evince dallo stesso titolo della questione: Utrum ultimo fine apprehenso in universali in via, voluntas necessario feratur in illum et velit ipsum.

11 Glorieux, "A propos de 'Vatic. lat. 1086", p. 27 e p. 34.

${ }_{12}$ "Proprius modus agendi nature ut nature respectu cuiuscumque obiecti est agere naturaliter et necessario, quia natura ut natura semper agit ex toto suo conatu, sed proprius modus agendi voluntatis respectu alicuius obiecti, puta respectu eius quod est ad finem non habens necessariam connexionem ad finem, est agere contingenter et libere" (Iacobus de Aesculo, Quodlibet, q. 1, citato in Alliney, "La ricezione", testo di nota 38).

${ }^{13}$ Come quello di Nicolò di Lira, anche il Commento di Giacomo d'Ascoli sopravvive in frammenti. 
come Giacomo difenda l'opposizione fra l'intelletto, considerato come un agente naturale, e la volontà libera. Dunque Giacomo mette in opera sia la teoria dei due principi, sia la dottrina dell'univocità della volontà, presupposti per la difesa della contingenza della fruizione. Tuttavia Giacomo afferma esplicitamente che le azioni volontarie libere e contingenti comuni alla volontà divina e alla volontà creata sono quelle rivolte ai beni parziali non necessariamente connessi con il fine, ma ciò comporta che la volontà umana agisca con necessità sia nei confronti del fine ultimo, sia nei confronti dei mezzi necessari per conseguirlo. Se questo è vero, Giacomo difende qui una dottrina tradizionale del secolo precedente, sostenuta già da Tommaso d'Aquino, ma anche da francescani come Gualtiero di Bruges. ${ }^{14}$

Secondo l'explicit di un codice oggi perduto Giovanni di Bassoles, uno dei discepoli preferiti di Scoto, lesse le Sentenze a Reims nel 1313. Bassoles, trattando della spirazione dello Spirito Santo, afferma che l'azione volontaria divina ad intra è caratterizzata dalla necessità, ribadendo, sulla scia di Scoto e di Ugo di Novocastro, che se la necessità libera è una perfezione della volontà, essa è in ogni caso distinta dalla necessità naturale perché ciò che deriva da principi opposti, quali natura e volontà, è di conseguenza opposto. Dunque, conclude Bassoles, la libertà in communi non si oppone alla necessità, ma si converte con la volontà stessa. ${ }^{15}$ Bassoles, al pari di Ugo di Novocastro e diversamente da Giacomo d'Ascoli, pare aver colto con precisione il senso della dottrina scotiana delle potenze attive, qui esposta con le debite articolazioni fra il piano delle differenze essenziali (natura/libertà) e quello delle differenze operative (necessario/contingente).

Tuttavia, quando affronta la questione della modalità della fruizione nei vari stati dell'uomo, il francescano non assume una posizione precisa. Secondo Giovanni, infatti, nessuna delle opinioni in discussione (necessità della fruizione in via o in patria; contingenza della fruizione in via o in patria) è dimostrabile né con argomenti di ragione né di fede, anche se egli è orientato ad ammettere la possibilità che la volontà del viator possa non volere il bene in generale. ${ }^{16}$ Questa mancata presa di posizione rimanda all'imbarazzato silenzio di Ugo di Novocastro, confermando così la dipendenza di Giovanni da Ugo già evidenziata in altri ambiti; ${ }^{17}$ ma testimonia ancora una volta la riluttanza dei francescani parigini ad aderire pienamente alla dottrina del Dottor Sottile.

Guglielmo di Alnwick legge le Sentenze a Parigi verosimilmente fra il 1313 e il 1315, dunque pochi anni dopo Giovanni di Bassoles. Dato che Alnwick è uno dei più importanti scotisti della prima generazione, il suo Commento rappresenta un fondamentale tassello per ricostruire lo sviluppo del dibattito sulla necessità della

14 Su questo punto si veda O. Lottin, Psychologie et morale aux XII et XIII siècles, I, Gembolux 1957, Capitolo 2.

Ioannes de Bassoles, In Sent., I, d. 10, q. un., art. 3, Parisiis 1516, ff. 100vb-101ra.

"[... videtur mihi dicendum quod neutra pars utriusque articuli de quo posite sunt opiniones est demonstrabilis nobis, nec ad aliquam earum potest haberi a nobis necessarium argumentum nec ex fide nec ex ratione" (Ioannes de Bassoles, In Sent., I, d. 1, q. 3, Parisiis 1516, f. 43va-b).

17 Cf. Volz, Die Lehre des Johannes de Bassolis, pp. 258-259. 
fruizione ${ }^{18}$. Affermando che la definizione formale univoca di libertà non esclude la necessità, Guglielmo espone la dottrina dell'univocità del concetto di volontà coerentemente alle intenzioni di Scoto, così come avevano fatto Ugo di Novocastro e Giovanni di Bassoles. ${ }^{19}$ Da questo presupposto Guglielmo sviluppa una teoria che ben si inserisce nel clima moderato dell'ambiente parigino, dato che egli sostiene la necessità della fruizione in patria e che, seguendo un'idea ormai diffusa, attribuisce tale necessità alla migliore conoscenza del fine ultimo che ora si mostra con chiarezza. ${ }^{20}$

$\mathrm{Ma}$, se si ammette con Scoto che la volontà agisce solo per motivi intrinseci, come si può giustificare il cambiamento del suo modo di agire da contingente a necessario? Alnwick afferma che non è necessario un cambiamento intrinseco alla potenza, ma è invece sufficiente un nuovo e diverso rapporto fra la potenza e l'oggetto per instaurare quella nova necessitas espressamente esclusa da Scoto. ${ }^{21}$ Alnwick dà così scarso rilievo all'infinità divina come condizione indispensabile perché la volontà possa agire con necessità, seguendo invece l'interpretazione moderata, legata alla tradizione francescano-agostiniana del secolo precedente, dell'univocità scotiana della volontà.

Quanto detto fin qui consente di valutare meglio la posizione dottrinale di Pietro Aureolo, teologo francescano di grande originalità, che giunge a Parigi nel 1316 con il proprio Commento alle Sentenze. ${ }^{22}$ Riguardo al tema in esame, Aureolo critica la teoria scotiana dell'opposizione fra natura/necessità e volontà/libertà in base alle irregolarità della natura, dato che "aliquando senes non canescunt". ${ }^{33}$ Aureolo ammette una definizione formale unica della libertà, valida per Dio e per l'uomo, ma rifiuta di collegare la libertà della volontà al dominio sui propri atti. È invece il

${ }_{18}$ Sulla datazione del Commento di Alnwick si veda G. Alliney, "Quaestiones de tempore o II Sent., d. 2, qq. 1-3? Chiarimenti sulla tradizione manoscritta di Guglielmo di Alnwick", Archivum Franciscanum Historicum 92/1-2 (1999), pp. 117-142: p. 138, dove si sostiene che probabilmente i principali codici che testimoniano il Commento di Alnwick riportano una seconda redazione stesa ad Oxford nel 1316. Sulla vita di Alnwick, e le edizioni delle sue opere, si veda Alliney, "E necessario amare Dio?", pp. 87-89.

19 Guillelmus de Alnwick, In Sent., I, d. 1, q. 1, n. 44, ed. Alliney, p. 120.

${ }_{20}^{20}$ "[... ipsa essentia divina in quantum est magis ostensa - quia nunc clare, prius obscure, licet hec ostensio sit voluntaria ex parte sui - ipsa tamen sic ostensa magis necessitat voluntatem ad agendum" (Guillelmus de Alnwick, In Sent., I, d. 1, q. 1, n. 78, ed. Alliney, p. 127).

${ }^{21}$ "[...] per hoc autem quod intellectus nunc videt obiectum, nihil novum est intrinsecum principio activo in fruitione; ergo nec nova necessitas agendi" (Ioannes Duns Scotus, Ordinatio, I, d. 1, p. 2, q. 2, n. 137, Id., Opera Omnia, Studio et cura Commissionis Scotisticae, Civitas Vaticana 1950ss., II, p. 92). Alnwick invece scrive che "[...] conceditur quod ibi sit nova necessitas, non tamen propter aliquid novum intrinsecum absolutum, nec hoc oportet, sed propter novam habitudinem potentie ad obiectum; nam ex nova habitudine necessaria creatur nova necessitas agendi" (Guillelmus de Alnwick, In Sent., I, d. 1, q. 1, n. 77, ed. Alliney, p. 127).

22 Per la datazione del commentario di Aureolo si veda Friedman, "The Sentences Commentary, 12501320", p. 82

${ }_{23}$ Petrus Aureoli, In I Sent., d. 1, sect. 8, n. 108, ed. E. M. Buytaert, New York-Louvain-Paderborn 1952, p. 448. 
piacere nell'agire che distingue l'azione libera da quella naturale: dunque anche gli animali sono liberi perché capaci di provare complacentia nelle proprie azioni. ${ }^{24}$

Come si vede, si tratta di opinioni assai diverse da quelle dei teologi di cui si è trattato; tuttavia, la conclusione raggiunta da Aureolo è simile: solo il fine ultimo "clare visus in patria" attrae la volontà tanto da necessitare il suo atto, e ciò è dovuto al cambiamento del modo di conoscerlo. Pietro Aureolo spiega questo mutamento tramite il passaggio dalla conoscenza astrattiva alla conoscenza intuitiva: ${ }^{25}$ il mutamento della contingenza in via nella necessità in patria assume la terminologia dei dibattiti gnoseologici. Al di là delle differenti argomentazioni, Aureolo non rappresenta dunque una reazione moderata alle tesi volontariste estreme diffuse a Parigi, ma si inserisce invece con continuità nel clima temperato presente nella capitale francese.

Per trovare un difensore del contingentismo scotiano bisogna attendere che Francesco di Meyronnes rediga nel 1320 il proprio Commento alle Sentenze. ${ }^{26}$ Analogamente ai francescani precedentemente citati, anche Francesco accoglie gli assunti di base della teoria della volontà di Scoto - l'opposizione formale fra potenza volitiva e intellettiva, dato che "una libere agit, alia naturaliter",; e l'esigenza di individuare una definizione della libertà in communi che non includa la contingenza. $^{28}$ A differenza di altri scotisti, tuttavia, egli inserisce questa distinzione all'interno della trattazione modale dell'essere, e limita la necessità del volere alla volontà divina in quanto infinita. ${ }^{29}$ Diversi modi di agire, come "necessario" e "contingente", possono coesistere nello stesso principio perché non si tratta di opposte ragioni formali, che sono invece "naturale" e "libero". In base a queste premesse, Meyronnes esclude che in qualunque modo sia conosciuto il fine ultimo la volontà lo voglia necessariamente..$^{31}$ La mancanza di necessità della volizione del fine in

24 "[... formalis ratio libertatis consistit in potentia ex actu complacentiae et delectationis. Actus enim complacentiae est formaliter liber; potentia autem, quae quicquid agit agit ex complacentia, dicitur libera; nec aliquid aliud exigitur ad rationem libertatis" (Petrus Aureoli, In I Sent., d. 1, sect. 8, n. 114, ed. Buytaert, p. 449). Riguardo agli animali: "Non est autem idem liberum et dominativum actus [...] sine dubio verum est, quod pueri et animalia voluntarium et spontaneum participant, et per consequens libertatem" (Petrus Aureoli, In I Sent., d. 1, sect. 8, n. 120, ed. Buytaert, p. 451).

25 "[...] voluntas immutabiliter elicit actum complacentiae circa Deum in patria clare visum" (Petrus Aureoli, In I Sent., d. 1, sect. 8, n. 122, ed. Buytaert, p. 452); "[..] fine ultimo qualitercumque citra intuitivam notitiam apprehenso, voluntas non de necessitate elicit actum complacentiae aut desiderii respectu illius" (Petrus Aureoli, In I Sent., d. 1, sect. 8, n. 130, ed. Buytaert, p. 457).

${ }^{26}$ Per la data del Commento di Francesco, indicata negli explicit di alcuni codici che lo trasmettono, si veda H. Roßmann, "Die Sentenzenkommentare des Franz von Meyronnes OFM", Franziskanische Studien 35 (1971), pp. 129-227.

27 Franciscus de Mayronis, In Sent., I, d. 10, q. 1, Venetiis 1520 (Frankfurt a. M. 1966), f. 56rb G.

28 "Dico quod libertas abstrahit a libertate arbitrii [... nam libertas stat cum necessitate ut abstrahit a libero arbitrio, et isto modo abstrahendo dicimus quod Spiritus Sanctus libere producitur, licet necessario, creatura autem non solum producitur libere absolute, sed libere libertate arbitrii" (Franciscus de Mayronis, In Sent., I, d. 10, q. 1, Venetiis 1520 (Frankfurt a. M. 1966), f. 56ra C).

29 Franciscus de Mayronis, In Sent., I, d. 10, q. 1, Venetiis 1520 (Frankfurt a. M. 1966), f. 56rb E.

Franciscus de Mayronis, In Sent., I, d. 10, q. 1, Venetiis 1520 (Frankfurt a. M. 1966), f. 56rb H.

31 "Finis clare visus non necessitat voluntatem, quia [... fruitio vel volitio contingenter causabitur, et posita poterit non poni" (Franciscus de Mayronis, In Sent., I, d. 1, q. 7, art. 3, Venetiis 1520 (Frankfurt a. M. 1966), f. 15ra-b D-E). 
patria deriva dalla costitutiva indeterminazione dell'essere finito, che trova a sua volta espressione nella naturale contingenza della causalità della volontà. ${ }^{32}$

Dopo le incertezze di Ugo di Novocastro e di Giovanni di Bassoles e l'opposizione di Guglielmo di Alnwick e di Pietro Aureolo, sembra questa la prima volta che il contingentismo scotiano venga apertamente difeso a Parigi. Ciò non implica, tuttavia, l'affermarsi diffuso della dottrina scotiana: negli stessi anni Francesco della Marchia nel suo Commento alle Sentenze (1319-1323) negherà ancora la contingenza della fruizione beatifica. ${ }^{33}$ A Parigi, dunque, la posizione di Duns Scoto resta minoritaria per tutto il primo quarto del XIV secolo: la maggioranza dei maestri in teologia considera la fruizione beatifica certo libera, ma al tempo stesso necessaria e non contingente.

\subsection{Oxford}

A differenza di quanto accade a Parigi, nell'ambiente francescano di Oxford la ricezione del pensiero di Scoto è lenta e contrastata. Nella città inglese è ancora forte l'influenza di Enrico di Gand, ${ }^{34}$ come è provato dalla posizione di Roberto Cowton, teologo francescano contemporaneo a Scoto, ed energico oppositore dello scozzese. ${ }^{35}$

Nel suo Commentario composto intorno al 1311-1312, Roberto imposta la questione della modalità della fruizione beatifica secondo criteri tradizionali, rifiutando l'opposizione scotiana fra natura e libertà, ed ammettendo di conseguenza la naturalità della necessità della fruizione. ${ }^{36}$ Roberto riporta a sostegno di tale conclusione un lungo passo della Summa di Enrico di Gand, dove

32 Francesco, trattando della volontà creata, afferma esplicitamente che "causalitas sua naturalis est contingens" (Franciscus de Mayronis, In Sent., I, d. 1, q. 8, Venetiis 1520 (Frankfurt a. M. 1966), f. 15va I).

${ }_{33}$ "[...] voluntas creata in obiectum beatificum veritate ostensum est magis inclinata naturaliter quam non inclinata. Ergo necessario elicit actum circa ipsum et contingenter" (Franciscus de Marchia, In Sent., I, d. 1, q. 6, Città del Vaticano, BAV, ms. Vat. lat. 1096, f. 15rb). Su Francesco della Marchia si veda R.L. Friedman, C. Schabel, "Francis of Marchia's Commentary on the Sentences: Question List and State of Research", Mediaeval Studies 63 (2001), pp. 31-105. E di prossima pubblicazione un mio studio sulla modalità della fruizione nel pensiero di questo autore.

34 Cfr. J.I. Catto, "Theology and Theologians 1220 - 1320", in J.I. Catto (ed.) The History of the University of Oxford, I, The Early Oxford Schools, Oxford 1984, p. 505, e W. J. Courtenay, Schools and Scholars in Fourteenth-century England, Princeton 1987, p. 187.

35 Roberto Cowton, francescano coetaneo di Scoto, ebbe notevole fama a Oxford, tanto che il suo Commento fu riprodotto in numerosi manoscritti e studiato fino al XV secolo. Sulla vita e le opere di Roberto cfr. H. Theissing, Glaube und Theologie bei Robert Cowton OFM, in BGPTM, XLII, 3, Münster 1969, pp. 4-12, in particolare il capitolo 7, § 23, pp. 206-220 sulla teoria della volontà. Giudicato in un primo tempo vicino a Scoto, Cowton è ora considerato un suo eclettico oppositore: si veda Courtenay, Schools and Scholars, p. 189.

${ }^{36}$ "Contra opinionem (scilicet non necesse est voluntatem frui fine ultimo) primo ostendo quod voluntas naturaliter vult finem ultimum in universali, et ex hoc concludo quod necessario vult finem ultimum in particulari, scilicet beatitudinem perfectam in obiecto quod natum est eam perfecte beatificare. Secundo declaro quod hec necessitas non repugnet eidem (ms.: eadem) libertati in hoc, quod voluntas vult finem de necessitate determinatione etiam determinante actum volendi ut egreditur a voluntate" (Robertus Cowton, In Sent., I, d. 1, q. 4, citato in Alliney, "Fra Scoto e Ockham", p. 261) 
il maestro secolare chiarisce che non è sufficiente escludere dalle azioni libere la necessità derivante dalla costrizione, poiché bisogna distinguere ancora la necessità che precede l'azione, e la determina - come nel caso degli agenti naturali -, dalla necessità che è concomitante con l'azione stessa, e che la potenza si autoimpone nella produzione dell'atto - come nel caso delle volizioni spontanee. $^{37}$

In definitiva, Cowton critica le tesi scotiane, ma utilizza argomenti genericamente volontaristi: diversamente da Parigi, dove il pensiero scotiano entra subito in circolazione negli ambienti universitari, a Oxford le innovazioni dottrinali di Scoto sono rifiutate a vantaggio di una eclettica adesione al volontarismo moderato.

Riccardo Conington, la cui influenza presso lo studio francescano della città inglese è ben nota, ${ }^{38}$ si mostra più originale. Nel suo primo Quodlibet, steso prima del $1310,{ }^{39}$ tre questioni sono dedicate alla modalità dell'azione volontaria. Nella prima di esse Conington afferma che la volontà agisce come una potenza naturale a causa della sua naturale tendenza al bene, e che questa naturalità implica la necessità della determinazione e della produzione dell'atto volontario, ma non della prosecuzione dell'azione. La volontà, poiché è determinata dalla propria intrinseca tendenza, agisce spontaneamente e senza subire violenza; tuttavia, tale azione non è libera, perché libertà e natura sono incompatibili. ${ }^{40}$ Nella seconda questione Riccardo precisa che la volontà creata nello stato attuale può sospendere qualsiasi atto verso il bene per l'incertezza della nostra conoscenza sensibile, che implica a sua volta un'imperfezione nell'oggetto in quanto conosciuto. Solo in Cielo la volontà potrà continuare l'azione con necessità, data la perfezione dell'oggetto, ovvero dell'Essenza divina conosciuta con chiarezza. ${ }^{41}$ Tale

37 Henricus de Gandavo, Summa quaestionum ordinariarum, a. 47, q. 5, Parisiis 1520, (New YorkLouvain-Paderborn 1953), II, ff. 27v - 28v, Y-Z.

${ }_{38}$ Egli fu giudicato dai contemporanei, al pari di Roberto Cowton, un discepolo di Enrico di Gand: vedi Doucet, "L'oeuvre scolastique", p. 420, nota 1, e S. D. Dumont, "William of Ware, Richard of Conington and the Collationes Oxonienses of John Duns Scotus", in L. Honnefelder, R. Wood, M. Dreyer (eds.), John Duns Scotus. Metaphysics and Ethics, Brill 1996, pp. 59-85: p. 68.

39 Vittorino Doucet ha dimostrato la paternità del Ouodlibet anonimo del codice Vaticano, Ottob. lat. 1126 e ha proposto una datazione che assume come termine post quem il 1306, inizio della reggenza di Riccardo Conington a Oxford, e come termine ante quem il 1310, data della sua nomina a ministro provinciale dell'ordine (V. Doucet, "L'oeuvre scolastique de Richard de Conington, O.F.M.", Archivum Franciscanum Historicum 29 (1936), pp. 396-442).

40 "Voluntas de se est in potentia essentiali ad operandum [...] ex quo patet quod voluntas de se non est naturaliter determinata ad aliquem actum. Sed voluntas affecta per impressionem in ea factam a bono in communi apprehenso est naturaliter determinata ad actum volendi ipsum subitum et indeliberatum" (Richardus de Conington, Quodlibet I, q. 6, citato in Alliney, "The Treatise on the Human Will", nota 118). Come Conington spiega poi, "licet sponte et non coacte velit, sed convenienter sue inclinationi, non tamen libere sed naturaliter; unde illa necessitas repugnat libertati" (Richardus de Conington, Quodlibet I, q. 8, citato in Alliney, "The Treatise on the Human Will", nota 118).

${ }_{41}$ "Ad principale dicendum quod [..] quod voluntas fertur in Deum contigenter in via, falsum est quantum ad exercitationem subitam actus indeliberati. Sed verum est quantum est ad continuationem eiusdem actus; et cum in via, ergo et in patria voluntas continuat actum contingenter, concedo, cum quo stat quod necessario continuat ipsum, ut patebit in proxima questione" (Richardus de Conington, Quodlibet I, q. 7, citato in Alliney, "The Treatise on the Human Will", nota 119). 
necessità è compatibile con la volontà perchè è imposta a se stessa dalla volontà medesima a causa dell'assenza di motivi per volere altro da Dio, e perciò essa può coesistere sia con la libertà della volontà, sia con la contingenza dell'atto, perché, se la volontà trovasse un oggetto più volibile di Dio conosciuto tramite l'intelletto del beato, potrebbe modificare la propria determinazione e volere tale oggetto. ${ }^{42} \mathrm{La}$ libertà della volontà è dunque spontaneità senza soggezione ad altre cause positive.

Le opinioni espresse da Conington mostrano indubbiamente, a differenza di quelle di Roberto Cowton, un forte carattere di originalità. L'esclusione dal novero delle azioni libere dell'atto prodotto naturalmente dalla volontà evidenzia l'influenza della lezione scotiana: natura e libertà si oppongono in quanto principi, e non possono mai coesistere. Riccardo, tuttavia, attribuendo alla volontà un'azione compiuta naturalmente e senza libertà, ammette implicitamente che la libertà non è costitutiva della volontà stessa; egli sembra piuttosto collegare stabilmente la libertà umana con una delle forme di contingenza attribuibili alle azioni volontarie. Anche se espressa con argomenti lontani da quelli scotisti (Conington non accenna all'univocità del concetto di "libertà", o alla differenza modale dell'essere), la conclusione è simile a quella che si è visto condivisa a Parigi: la necessità in patria deriva dalla perfezione dell'oggetto voluto.

Si è detto come a Parigi Guglielmo di Alnwick assuma una posizione che nella sua conclusione generale non è diversa da quella di Conington, dato che la necessità della fruizione è dimostrata in base alla mancanza di alcun motivo per volere altro. Alnwick ritorna in Inghilterra intorno al 1316, e fornisce così ulteriore materiale a sostegno della tesi della necessità della fruizione in patria. Nello stesso periodo vengono conosciute ad Oxford anche le tesi di Pietro Aureolo. ${ }^{43}$ Nei primi tre lustri del secolo, dunque, sono contemporaneamente presenti a Oxford sia influenze gandiane (Cowton), sia autonome elaborazioni di alcuni punti del pensiero di Scoto (Conington), sia infine gli ultimi sviluppi del dibattito parigino, di ambiente scotista (Alnwick) o generalmente francescano (Aureolo). Ciò che accomuna queste tendenze è l'ammissione della necessità del volere del beato in patria. A questo riguardo vale notare che anche le Collationes Oxonienses di Duns Scoto sostengono una dottrina simile, per la quale l'assenza di motivi in contrario fissa la volontà del beato nella fruizione beatifica, ma recenti studi hanno messo in dubbio l'attribuzione a Scoto di tali questioni sulle volontà, supponendo invece che si tratti di registrazioni di discussioni avvenute nel convento francescano di Oxford in assenza del maestro. ${ }^{44}$ Se apocrife, le Collationes confermerebbero

42 "Necessitas autem qua omnis voluntas videntis Deum continuat necessario velle Deum non est immutabilitas nature voluntatis eius, sicut in Deo, nec est ei illata ab aliqua causa positiva. Manet tantum a causa privativa que est defectus cur, ut supra ostensum est, et quia cause privative non est effectus positivus [... quia posito per impossibile aliquod appetibile cuius bonitatem non gustasset modo eminentiori volendo Deum apprehensum [di] per intellectum, voluntas haberetur cur posset prediligere ipsum [...] nec autem esset hoc impossibile propter defectum cur" (Richardus de Conington, Quodlibet I, q. 8, citato in Alliney, "The Treatise on the Human Will", nota 120).

43 Sulla diffusione del pensiero di Aureolo in Inghilterra si veda Courtenay, Schools and Scholars, p. 189; sul rapporto fra Alnwick e Aureolo si veda Alliney, "E necessario amare Dio?", pp. 108-110.

4 Si veda Alliney, "The Treatise on the Human Will". 
dunque la resistenza dell'ambiente minorita oxoniense alla diffusione delle dottrine genuinamente scotiane.

Solo alla fine del secondo decennio del '300 Giovanni di Reading, Scoti discipulus et sequax valentissimus ${ }^{45}$ in una lunga questione del suo Commento alle Sentenze ribadisce la conclusione di Scoto riguardo alla contingenza della fruizione beatifica. $^{46}$ La questione, riportando solamente opinioni di teologi francescani, conferma sia il successo dell'ordine a Oxford a scapito dei predicatori, ${ }^{47}$ sia la varietà delle posizioni all'interno della speculazione minorita.

Reading si oppone anzitutto ai teologi inglesi come Roberto Cowton e Riccardo Drayton che, legati alle opinioni tradizionali, sostengono la necessità, quantomeno in patria, della fruizione del sommo bene. Drayton, a differenza di Cowton, concorda con Riccardo di Conington nell'attribuire la necessità della fruizione beatifica alla mancanza di motivo per la volontà di allontanarsi da un oggetto perfettissimo ${ }^{48}$ Ma il principale obiettivo polemico di Reading è Pietro Aureolo, oppositore di Scoto non solo nelle conclusioni generali, ma anche e soprattutto nel propugnare una concezione di libertà, colta nella dilettazione per l'atto volontario, che mina alla base il collegamento, fondamentale nel pensiero scotiano, fra aspetto libero e aspetto potestativo della volontà.

Aureolo critica l'assoluta intrinsecità del modo d'agire della volontà, sostenendo che la maggior perfezione dell'oggetto può rendere necessaria la volizione, dato che il modo d'agire non è determinato solo dalla potenza, ma dal rapporto fra la potenza e l'oggetto, ex concursu amborum. ${ }^{49}$ Reading ribatte che, indipendentemente dalla perfezione dell'oggetto, è la stessa libertà che può essere motivo del sottrarsi della volontà alla fruizione. ${ }^{50}$ Egli argomenta che l'univocità del concetto di "libertà" non è un'unità reale, e quindi non implica sempre un medesimo modo d'agire, dato che Dio vuole ogni cosa con immutabilità, ma non così la creatura, che agisce invece sempre con contingenza ${ }^{51}$ a causa della propria limitatezza. ${ }^{52}$ Reading, tuttavia, non pone sempre una chiara distinzione fra

45 Adam Wodeham, Lectura Oxoniensis, I, d. 1, q. 12, Civitas Vaticana, BAV, ms. Vat. Lat. 955, f. 70v Il Commento alle Sentenze di Reading risale al 1317-1319. Per una ricostruzione della vita di Giovanni si veda S. J. Livesey, Theology and Science in the Fourteenth Century. Three Questions on the Unity and Subalternation of the Sciences from John of Reading's Commentary on the Sentences, Leiden-New York-København-Köln 1989, pp. 3-7.

47 Courtenay, Schools and Scholars, pp. 181-185.

48 "[...] a parte obiecti deficit cur voluntas possit se suspendere ad actum volendi" (Opinione attribuita a Drayton in Ioannes de Reading, In Sent., I, d. 1, q. 6, n. 220, ed. Alliney, p. 342).

49 Petrus Aureoli, In I Sent., d. 1, sect. 8, n. 109, ed. Buytaert, p. 448, citato da Reading in In Sent., I, d. 1, q. 6, n. 163, ed. Alliney, p. 328.

50 "Contra illud quod additur, quod non potest non velle finem, quia non habet cur in obiecto respectu aversionis; [..] libertas, sive experientia libertatis, potest esse cur possumus avertere" (Ioannes de Reading, In Sent., I, d. 1, q. 6, n. 228, ed. Alliney, p. 343). Si vedano anche i nn. 238-239 alle pp. 344-345.

51 "[... voluntas Dei et nostra non habent unitatem in re, quamvis habeant univocationem in conceptu; et ex hoc non sequitur idem modus volendi illa que volunt, quia Deus immutabiliter vult quicquid vult, voluntas nostra non" (Ioannes de Reading, In Sent., I, d. 1, q. 6, n. 216, ed. Alliney, p. 341).

52 "Quamvis ergo non esset de ratione libertatis in communi posse suspendere actum, tamen de ratione libertatis, in quantum limitata, est posse suspendere actum respectu cuiuscumque obiecti divisim" (Ioannes de Reading, In Sent., I, d. 1, q. 6, n. 273, ed. Alliney, p. 353). 
principi (natura e libertà) e modi di agire (necessità e contingenza), e tende a identificare libertà umana e contingenza, correndo così il rischio di ridurre la teoria scotiana a una forma di contingentismo. ${ }^{53}$ Nonostante queste incertezze teoriche, Reading resta tuttavia importante per essere il primo teologo ad Oxford a tenere in debito conto la metafisica ontologica di Scoto.

Nel primo ventennio del secolo la soluzione scotista pare essere difesa unicamente da Reading, al quale si oppongono non solo i teologi che tendono a separare la libertà dalla contingenza, ma anche da chi, al contrario, è pronto ad identificare i due concetti. Ė la via che viene imboccata da Guglielmo di Ockham, il quale stende il proprio Commento alle Sentenze contemporaneamente a Reading. Ockham, in polemica con le complicate distinzioni terminologiche scotiane, afferma infatti che la libertà non è costitutiva della volontà, ma piuttosto è un termine connotativo che denota la potenza e connota che qualcosa può essere fatto contingentemente da questa. Il Venerabilis inceptor sostiene che la volontà umana mantiene la capacità di non volere Dio, pur conosciuto con chiarezza, perché essa deve essere sempre in grado di obbedire ad un eventuale ordine di Dio che le imponga di odiarLo, ${ }^{54}$ ma dietro questa apparente convergenza si cela una differenza di fondo: Scoto deduce la contingenza della fruizione dall'analisi del concetto di libertà che, a priori, distingue la volontà dagli altri principi, mentre Ockham può attribuire la libertà alla volontà solo a posteriori, dopo averne mostrato la contingenza nella fruizione. ${ }^{55}$ Con questa tesi Ockham si pone fuori dal dibattito teso a cogliere la specificità della volontà in quanto libera: nella impostazione occamiana "libero" diviene una specie di etichetta, applicabile a qualunque atto compiuto contingentemente. ${ }^{56}$ La diffusione delle dottrine occamiste decreta una momentanea crisi dello scotismo inglese: più ancora che a Parigi, il volontarismo scotiano trova a Oxford solo occasionali difensori, a riprova della scarsa fortuna della rivoluzionaria concezione della libertà del teologo scozzese in Inghilterra. I teologi tradizionali, infatti, ritengono la fruizione beatifica libera ma necessaria, mentre gli occamisti, all'opposto, la reputano contingente, ma proprio per questo non libera.

53 Si veda Alliney, "Fra Scoto ed Ockham", pp. 280ss.

${ }^{54} \quad \grave{\mathrm{E}}$ il famoso argomento dell'odium Dei, per il quale "Deus potest praecipere quod voluntas creata odiat eum, igitur voluntas creata potest hoc facere". (Guillelmus de Ockham, IV Sent., d. 14, citato in A. Ghisalberti, Introduzione ad Ockham, Roma-Bari 1976, p. 62, dove si inquadra il senso del passo nella totalità del pensiero occamiano). La contingenza della volontà è basata dunque sull'onnipotenza divina.

55 Ockham imputa a Scoto un'errata comprensione del significato di "libertà": "Imaginatur enim ac si libertas esset aliquid unum reale distinctum aliquo modo ex natura rei a voluntate, [...] quod tamen non est verum. Sed est unum nomen connotativum importans ipsam voluntatem [...] connotando aliquid contingenter posse fieri ab eadem [...] dico quod non est sic condicio intrinseca potentiae [...]. Concedo quod voluntas est potentia libera, non tamen respectu cuiuscumque, sed respectu illius ad quod contingenter se habet" (Guillelmus de Ockham, I Sent., d. 10, q. 1, in Id., Opera Theologica, III, ed. G. J. Etzkorn, St. Bonaventure, N. Y., 1977, pp. 343-345).

56 Per questo Clark ritiene che "the metaphysics of freedom taught by Ockham seems rustic. Contingency is a spark without flint and steel, a surd within a predictable melody" (D. W. Clark, "Ockham on Human and Divine Freedom", Franciscan Studies 38 (1978), p. 147). 